\title{
Turf-Toe traumático: diagnóstico desafiante y tratamiento controvertido
}

\author{
Traumatic Turf-Toe: challenging diagnosis and controversial treatment
}

\author{
Gómez-Carlín LA, * Yáñez-Solís GK, ${ }^{\ddagger}$ Patiño-Fernández JP,$\$$ \\ Ramírez-Gómez VJ, ${ }^{\uparrow}$ Valdez-Cueva V," Ortega-Orozco R**
}

Antiguo Hospital Civil de Guadalajara «Fray Antonio Alcalde».

\begin{abstract}
RESUMEN. Introducción: Turf-Toe incluye una gran variedad de lesiones traumáticas de la articulación metatarsofalángica del primer dedo del pie (MTF1). Es una lesión potencialmente grave y no existe consenso para el manejo quirúrgico. El objetivo de este reporte fue describir el caso de un paciente con lesión Turf-Toe traumática grado III con fractura sesamoidea tratado quirúrgicamente. Caso clínico: Masculino de 24 años con caída de motocicleta con hiperflexión de la articulación MTF1 presentó dolor, edema y limitación funcional; las radiografías mostraron edema de tejidos blandos, desplazamiento lateral de sesamoideo lateral con fractura. Se estableció el diagnóstico de Turf-Toe traumático. En el ultrasonido de la articulación MTF1, se demostró rotura de la placa plantar y ligamentos colaterales. Se manejó quirúrgicamente con reducción del sesamoideo y reparación de la placa plantar. La evolución postoperatoria fue satisfactoria; a las ocho semanas el paciente fue valorado con escala AOFAS de antepié obteniendo 82 puntos, SF12 con $87 \%$ y EVA de 2 y retornó a sus actividades cotidianas. Conclusión: Describimos un paciente con lesión Turf-Toe traumática grado III, no relacionada con la práctica deportiva. Primer reporte en la literatura del uso del
\end{abstract}

ABSTRACT. Introduction: Turf-Toe includes a wide variety of traumatic injuries of the metatarsophalangeal joint of the first toe (MTF1). It is a potentially severe injury and there is no consensus for surgical management. The objective of this case report was to describe a patient with traumatic Turf-Toe injury grade III with a sesamoid fracture treated surgically. Clinical case: 24 -year-old male with motorcycle fall with hyperflexion of the MTF1 joint, presented pain, edema and functional limitation; the radiographs showed soft tissue edema, lateral displacement of lateral sesamoid with fracture. Traumatic Turf-Toe was established. The ultrasound of the MTF1 joint showed breakage of the plantar plate and collateral ligaments. Patient was surgically managed with sesamoid reduction and plantar plate repair; postoperative evolution was satisfactory. At 8 weeks the patient was assessed with the AOFAS forefoot scale, obtaining 82 points, SF-12 with $87 \%$ and VAS of two and returned to his daily activities. Conclusion: We describe a patient with traumatic Turf Toe grade III injury, not related to sports practice; first report in the literature of the use of ultrasound in the evaluation of patients with lesion of the metatarso-phalangic joint of the first toe.

\footnotetext{
* Especialista en Traumatología y Ortopedia, Alta Especialidad de Cirugía de Pie y Tobillo. Jefe de la Clínica de Pie y Tobillo. Medyarthros Medicina Deportiva y Artroscopía. Profesor Titular de Curso de Alta Especialidad de Cirugía de Pie y Tobillo, Centro Universitario de Ciencias de la Salud. Universidad de Guadalajara.

${ }^{\ddagger}$ Residente de tercer año en Traumatología y Ortopedia. Antiguo Hospital Civil de Guadalajara «Fray Antonio Alcalde».

${ }^{\S}$ Residente de cuarto año en Traumatología y Ortopedia. Antiguo Hospital Civil de Guadalajara «Fray Antonio Alcalde».

" Especialista en Traumatología y Ortopedia, Alta Especialidad de Cirugía de Pie y Tobillo. Médico adscrito a la Clínica de Pie y Tobillo, Medyarthros Medicina Deportiva y Artroscopía.

"Especialista en Traumatología y Ortopedia; adiestramiento en Artroscopía y Medicina Deportiva. Antiguo Hospital Civil de Guadalajara «Fray Antonio Alcalde».

** Director General Medyarthros Medicina Deportiva y Artroscopía. Jefe del Servicio de Artroscopía y Medicina Deportiva Antiguo Hospital Civil de Guadalajara «Fray Antonio Alcalde». Profesor asociado de Traumatología y Ortopedia del Centro Universitario de Ciencias de la Salud. Universidad de Guadalajara.
}

Correspondencia:

Dr. Luis A Gómez-Carlín

Av. Ignacio L Vallarta Núm. 3060, Col. Ladrón de Guevara, C.P. 44600, Guadalajara, Jalisco, México.

E-mail: luiscarlin@medyarthros.com

Citar como: Gómez-Carlín LA, Yáñez-Solís GK, Patiño-Fernández JP, Ramírez-Gómez VJ, ValdezCueva V, Ortega-Orozco R. Turf-Toe traumático: diagnóstico desafiante y tratamiento controvertido. Acta Ortop Mex. 2020; 34(5): 319-323. https://dx.doi.org/10.35366/97996

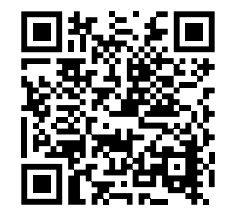


ultrasonido en la evaluación de pacientes con lesión de la articulación metatarso-falángica del primer dedo del pie.

Palabras clave: Metatarsofalángica, postraumática, placa plantar, hueso sesamoideo.

\section{Introducción}

La descripción original de la lesión Turf-Toe fue realizada por Bowers y Martin en 1976; ${ }^{1}$ esta fue identificada originalmente en jugadores de fútbol americano asociada al pasto artificial. ${ }^{2}$ En la actualidad, el término Turf-Toe incluye una gran variedad de lesiones traumáticas de la articulación metatarsofalángica del primer dedo del pie (MTF1) que son similares en el mecanismo y localización pero que resultan de actividades deportivas diferentes o traumatismos no relacionados con alguna actividad deportiva. El aumento de estrés sobre el lado medial del pie predispone a la lesión de la articulación MTF1 por la hiperextensión forzada que afecta al tejido conectivo y la placa plantar; además, puede acompañarse de fractura sesamoidea de la articulación MTF1, ${ }^{2,3}$

La incidencia de la lesión Turf-Toe es desconocida debido a que existe subregistro de ésta; el diagnóstico requiere alto grado de sospecha: ${ }^{4}$ la evaluación clínica permite identificar la lesión, su localización y severidad. ${ }^{5}$ Se clasifica en tres grados: los grados I y II se consideran lesiones leves sin alteraciones en radiografías simples; en contraste, la lesión grado III se presenta clínicamente con dolor severo a la palpación, edema y equimosis marcada con limitación al movimiento y signo de Lachman positivo que demuestra inestabilidad o hiperflexión pasiva forzada de la articulación MTF 1., Las lesiones asociadas a Turf-Toe grado III incluyen: fractura sesamoidea, diástasis de sesamoideos bipartitos, impactación articular metatarsiana y/o migración sesamoidea. En radiografías simples se pueden encontrar alguno(s) de los siguientes hallazgos: fractura-avulsión de falange proximal, fractura de sesamoideo, migración proximal de sesamoideo y/o luxación de la articulación MTF $1 .{ }^{6}$

Turf-Toe es una lesión potencialmente grave que puede provocar secuelas como dolor, debilidad para empujar con el MTF1, rigidez, deformidad, desarrollo de artrosis con impacto funcional en las actividades deportivas y de la vida diaria. ${ }^{5}$

El propósito del manejo inicial de la lesión Turf-Toe es eliminar el dolor y disminuir el edema con reposo, hielo, compresión, elevación de la extremidad y medicamentos antinflamatorios; ${ }^{8}$ este manejo conservador y la inmovilización, en promedio de ocho semanas, es el tratamiento indicado en las lesiones grado I y II. En contraste, la información publicada aún es insuficiente para establecer el manejo estándar de la lesión grado III; ${ }^{5}$ se ha demostrado que el manejo quirúrgico de ésta presenta evolución clínica satisfactoria con menor tiempo de inmovilización comparado con el manejo conservador. Aunque actualmente no existe
Keywords: Metatarsophalangeal, post-traumatic, plantar plate, sesamoid bone.

consenso de las indicaciones quirúrgicas, se considera a la cirugía como el tratamiento de elección en Turf-Toe grado III. ${ }^{4}$ El objetivo de este reporte fue describir el caso de un paciente con lesión Turf-Toe traumática grado III con fractura sesamoidea tratado quirúrgicamente.

\section{Caso clínico}

Paciente masculino de 24 años de edad, previamente sano; al estar conduciendo su motocicleta sobre terreno rocoso sufrió caída de la misma y ésta se impactó sobre su talón derecho ocasionando hiperflexión de la articulación MTF1. El paciente escuchó un «chasquido» e inmediatamente presentó dolor, edema y limitación para la flexión dorsal de la MTF1, así como limitación funcional a la marcha. Acudió a la Clínica de Pie y Tobillo, cuatro días posteriores al traumatismo, con edema de la articulación MTF1 del pie derecho (Figura 1). Sus radiografías, anteroposterior (AP) y oblicua, mostraron edema de tejidos blandos en la articulación MTF1, desplazamiento lateral de sesamoideo lateral con fractura sobre su tercio proximal sin datos de conminución o luxación: se estableció el diagnóstico de Turf-Toe de pie derecho (Figura 2). En el ultrasonido Doppler de la articulación MTF1 se demostró rotura de la placa plantar y de los ligamentos colaterales. Se identificó
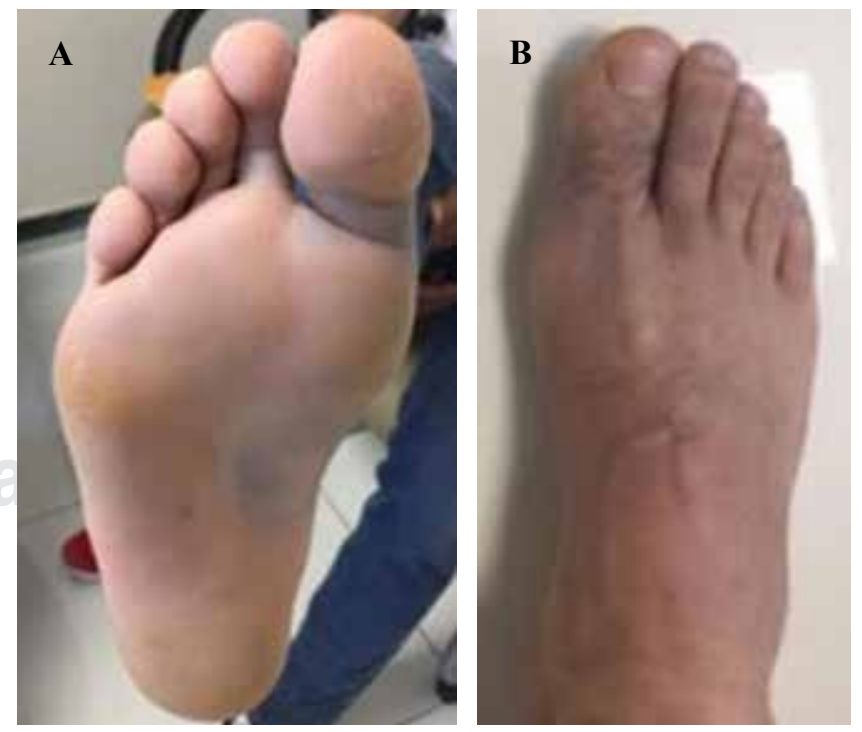

Figura 1: A) Se observaron hematomas en la articulación MTF1 y el arco medial con probable afectación de la placa plantar. B) Se encontró edema en la articulación MTF1 con hematoma que se extiende medial y dorsalmente hasta la diáfisis del metatarso. 

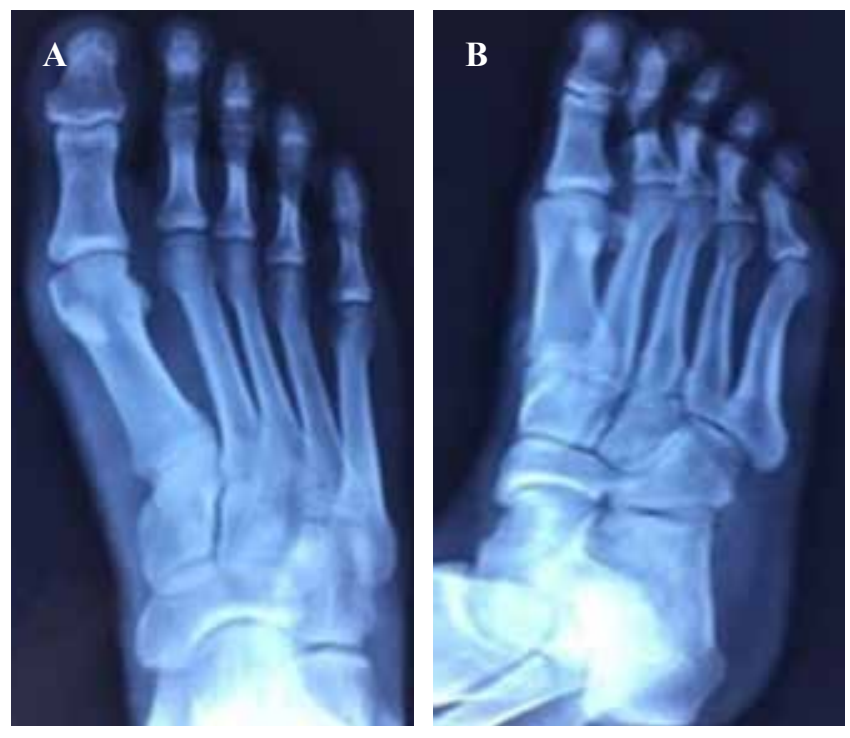

Figura 2: A) Radiografía anteroposterior de pie que mostró edema sobre la articulación MTF1 y desplazamiento lateral del sesamoideo lateral. B) Se observó fractura en el tercio proximal del sesamoideo lateral sin datos de conminución o luxación: se estableció el diagnóstico de lesión Turf-Toe.

trazo de fractura del sesamoideo lateral con desplazamiento lateral de $8 \mathrm{~mm}$. Se observó incremento de la vascularidad periférica (Figura 3).

El manejo inicial consistió en colocación de férula tipo Walker; se indicó apoyo diferido. Posteriormente, se procedió al manejo quirúrgico con reducción del sesamoideo y reparación de la placa plantar. El abordaje fue medial sobre la articulación MTF1; se encontró íntegro el trayecto e inserciones del abductor y flexor corto del primer dedo. Al realizar la disección profunda, se encontró rotura de la cápsula articular, de la placa plantar y del ligamento colateral lateral. Asimismo, se identificó el trazo de fractura longitudinal sobre el borde lateral del sesamoideo lateral (Figura 4). Se realizaron perforaciones del sesamoideo lateral con clavo Kirshner $1.8 \mathrm{~mm}$ y se procedió a la re- paración con sutura no absorbible número 2-0. Se realizó plastía con reparación directa de la placa plantar y del ligamento colateral lateral con sutura no absorbible número 2-0; se realizó cierre de la cápsula articular con puntos simples (Figura 5 A-C). A continuación, se procedió al cierre por planos y control fluoroscópico para confirmar la alineación correcta del sesamoideo lateral; se colocó vendaje al hallux y vendaje algodonado de Robert Jones.

La evolución postoperatoria fue satisfactoria; se realizaron radiografías de pie derecho (AP, oblicua y lateral) que mostraron alineación del sesamoideo lateral. El paciente fue egresado al día siguiente de la cirugía; con apoyo diferido de la extremidad y uso de zapato rígido hasta la semana cuatro. El manejo se continuó con rehabilitación para el fortalecimiento y recuperación de arcos de movimiento con carga de peso gradual y a tolerancia. A las ocho semanas posteriores de la cirugía el paciente fue valorado con escala AOFAS de antepié obteniendo 82 puntos, SF-12 con $87 \%$ y EVA de 2; retornó a sus actividades cotidianas sin limitación funcional.

\section{Discusión}

En este reporte de caso, se presenta un paciente con lesión Turf-Toe grado III no relacionada con la práctica deportiva; el diagnóstico se realizó apoyado del uso de ultrasonido y Rayos X y el tratamiento fue quirúrgico con evolución satisfactoria; el paciente retornó a sus actividades cotidianas a las ocho semanas. Éste es nuestro primer reporte en México de lesión Turf-Toe traumático.

No existen estudios aleatorios y con cegamiento para establecer el tratamiento estándar de la lesión Turf-Toe; ${ }^{4}$ el propósito del manejo quirúrgico es restaurar la anatomía y la estabilidad de la articulación MTF1. ${ }^{2}$ La American Academy of Orthopaedic Surgeons (AAOS) ha propuesto el tratamiento quirúrgico en los casos con rotura severa del complejo plantar, fractura de sesamoideos, inestabilidad vertical de la articulación MTF1, fragmento de sesamoideo intraarticular y aumento de la deformidad dorsal. Aun
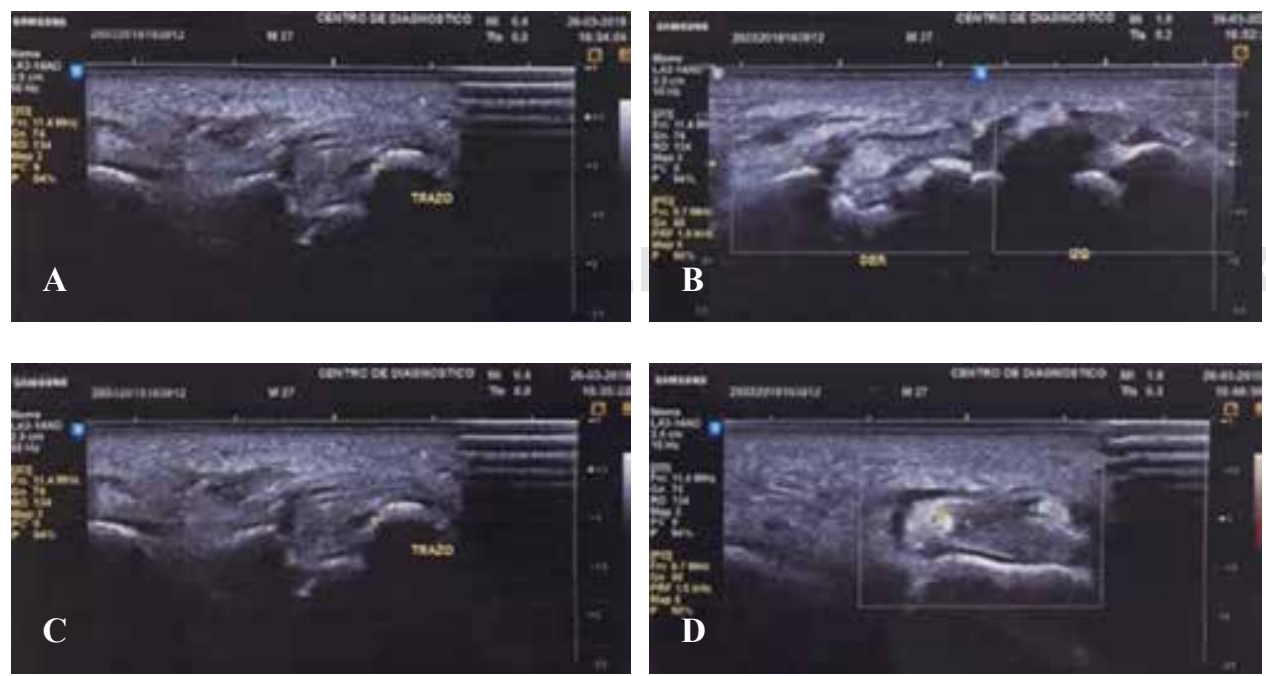

Figura 3:

A y B) El ultrasonido de la articulación MTF1 identificó el trazo de fractura en la base del primer metatarsiano del pie derecho con incremento $(8 \mathrm{~mm})$ en la separación del hueso sesamoideo interno. C y D) Mientras que la separación en el lado contralateral es normal (4 mm). 


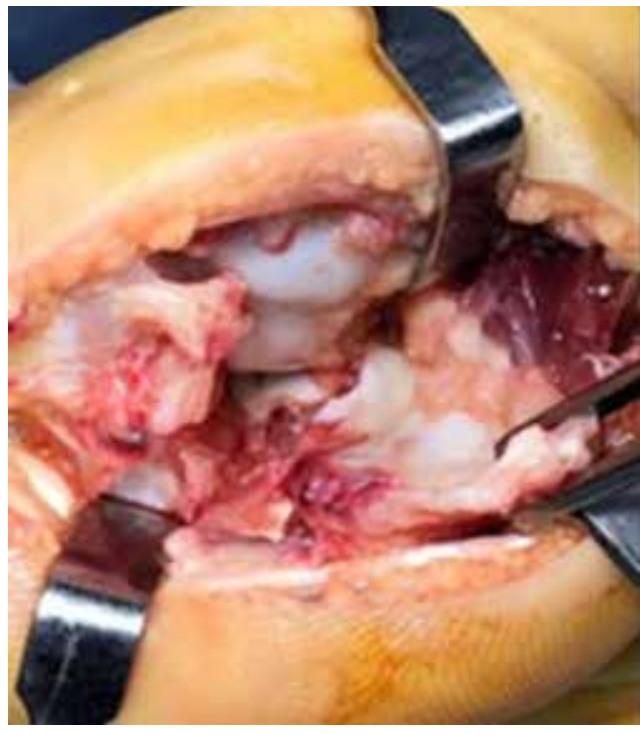

Figura 4: Rotura de la cápsula articular, de la placa plantar y del ligamento colateral lateral. Trazo de fractura longitudinal sobre el borde lateral del sesamoideo lateral.

cuando no existe consenso entre los distintos autores de la indicación del manejo quirúrgico, se recomienda promover la aplicación de los criterios propuestos por la AAOS y evitar criterios adicionales. ${ }^{9}$ En un estudio en 24 pacientes con Turf-Toe traumático y seguimiento a mediano plazo de 4.5 años (promedio 2-17 años) se presentaron dos casos con lesión grado I, 8 casos con lesión grado II y 14 con lesión grado III. Las lesiones grado I y II se manejaron conservadoramente, mientras que las de grado III fueron manejadas con cirugía. La evaluación clínica y funcional de 10 pacientes con manejo conservador mostró escala AOFAS inicial de 51 y en el seguimiento se alcanzaron 84 puntos. En comparación, en 14 pacientes con lesión grado III tratados con cirugía, se encontró AOFAS 36 y 81 en período prey postoperatorio, respectivamente; ${ }^{7}$ el resultado del estudio es comparable con este reporte de caso; dicho paciente con lesión Turf-Toe traumática grado III fue tratado con manejo quirúrgico y presentó evolución favorable con buenos resultados clínicos y funcionales con puntuación de 82 en la escala AOFAS.

Las lesiones de los huesos sesamoideos representan un reto debido a su pobre vascularidad y cicatrización. ${ }^{8} \mathrm{La}$ función de los sesamoideos es servir como fulcro para incrementar la fuerza en la flexión plantar que ofrece ventaja mecánica al tendón del flexor corto del primer dedo del pie y da soporte a la cabeza metatarsal; ;,2 la articulación MTF1 es estabilizada a los lados por los ligamentos colaterales y dorsalmente por la expansión del tendón. Se ha reportado con mayor frecuencia el desplazamiento medial del hueso sesamoideo relacionado con el soporte de la mayor carga de peso en la articulación MTF $1 .{ }^{10}$ En la literatura existen diferentes estrategias de manejo de la fractura de hueso sesamoideo, como fijación interna, curetaje, injerto óseo, entre otras. ${ }^{11}$ En este reporte de caso, se demostró desplazamiento lateral de sesamoideo lateral con fractura sobre su tercio proximal, sin datos de conminución o luxación, que fue fijada con sutura no absorbible número 2-0.

No existe consenso respecto a los estudios de gabinete ideales para el diagnóstico de Turf-Toe ${ }^{8}$ La resonancia magnética nuclear (RMN) del pie es comúnmente utilizada, principalmente en la lesión grado II o III: ${ }^{2}$ permite establecer el grado al identificar lesiones óseas, articulares y de tejidos blandos. ${ }^{2,4,5}$ La RMN es útil cuando se sospecha clínicamente el diagnóstico de lesión Turf-Toe y los hallazgos radiográficos son normales. En este reporte de caso, el diagnóstico se estableció con radiografía AP y oblicua de pie; además, se realizó ultrasonido Doppler para la evaluación de tejidos blandos de la articulación MTF1 que mostró rotura de la placa plantar y de los ligamentos colaterales; estos hallazgos sonográficos no se identificaron en radiografías simples. En nuestro paciente no se realizó RMN del pie debido a la limitación en la disponibilidad del estudio en la institución. El estudio ultrasonográfico de la articulación MTF1 fue útil para establecer la severidad de la lesión y la indicación del tratamiento quirúrgico en este reporte de caso con TurfToe traumático.
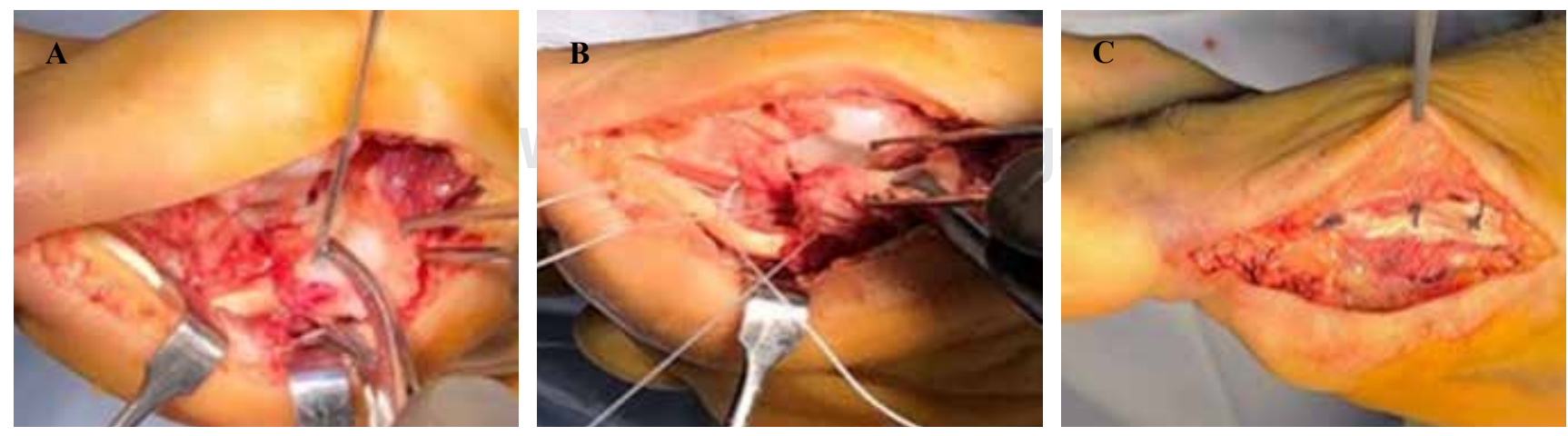

Figura 5: A) Perforaciones del sesamoideo lateral con clavo Kirschner 1.8 mm, reparación con sutura no absorbible número 2-0. B) Plastía con reparación directa de la placa plantar y del ligamento colateral lateral con sutura no absorbible número 2-0. C) Cierre de la cápsula articular con puntos simples. 


\section{Conclusión}

En este reporte se describe el caso de un paciente con lesión Turf-Toe traumática grado III, no relacionada con la práctica deportiva; el manejo fue quirúrgico con buena evolución clínica y funcional (AOFAS 82). Se utilizó el ultrasonido de la articulación MTF1 en la evaluación diagnóstica; se considera que el ultrasonido de la articulación MTF1 puede ser de utilidad en pacientes con sospecha de lesión Turf-Toe, cuando no está disponible la resonancia magnética.

\section{Agradecimientos}

Los autores agradecen a la doctora Ana María Contreras de la Unidad de Investigación de Desarrollo Tecnológico (UNIDET) por su asesoría científica en la redacción de este caso clínico.

\section{Referencias}

1. Bower KD Jr, Martin RB. Turf-Toe: a shoe-surface related football injury. Med Sci Sports. 1976; 8(2): 81-3.

2. McCormick JJ, Anderson RB. Turf-Toe: anatomy, diagnosis, and treatment. Sports Health. 2010; 2(6): 487-94.
3. George E, Harris AHS, Dragoo JL, Hunt KJ. Incidence and risk factors for turf toe injuries in intercollegiate football: data from the national collegiate athletic association injury surveillance system. Foot Ankle Int. 2014; 35(2): 108-15.

4. Hainsworth L, McKinley J. The management of turf toe: a systematic review. Baltic J Sport Health Sci. 2018; 110(3): 25-37.

5. Mason LW, Molloy AP. Turf-Toe and disorders of the sesamoid complex. Clin Sports Med. 2015; 34(4): 725-39.

6. Anderson RB. Turf Toe injuries of the hallux metatarsophalangeal joint. Techniques in Foot Ankle Surgery. 2002; 1(2): 102-11.

7. Nery C, Fonseca LF, Gonclaves JP, Mansur N, Lemos A, Maringolo L, et al. First MTP joint instability: expanding the concept of "TurfToe" injuries. Foot Ankle Surg. 2020; 26(1): 47-53. https://doi. org/10.1016/j.fas.2018.11.009.

8. Marchetti DC, Chang A, Ferrari M, Clanton TO. Turf Toe: 40 years later and still a problem. Operative Technique in Sports Medicine. 2017; 25(2): 99-107.

9. Waldrop N, Smith K. Surgical outcomes of severe Turf-Toe injuries in high-level football players. Foot Ankle Orthopaedics. 2017; 2(3): doi: $10.1177 / 2473011417$ S000402.

10. Kubitz ER. Athletic injuries of the first metatarsophalangeal joint. $J$ Am Podiatr Med Assoc. 2003; 93(4): 325-32.

11. Smith K, Waldrop N. Operative outcomes of grade 3 Turf-Toe injuries in competitive football players. Foot Ankle Int. 2018; 39(9): 1076-81. doi: $10.1177 / 1071100718775967$.

Conflicto de intereses: No hay conflicto de intereses qué declarar. 